Australasian Journal of LogiC

\title{
Relevance Logic: Problems Open and Closed
}

\author{
Alasdair Urquhart \\ Department of Computer Science \\ University of Toronto *
}

January 22, 2016

\begin{abstract}
I discuss a collection of problems in relevance logic. The main problems discussed are: the decidability of the positive semilattice system, decidability of the fragments of $\mathbf{R}$ in a restricted number of variables, and the complexity of the decision problem for the implicational fragment of R. Some related problems are discussed along the way.
\end{abstract}

\section{Introduction}

This paper is a lightly edited version of a talk presented at the Summer of Logic in Vienna in July 2014. The aim of the talk was to present a handful of unsolved problems in the area of relevance logic, as well as to report the very recent solution of a long-standing open problem. The selection obviously reflects my own interests; I encourage other researchers to present their own favourite problems. If the health of an area of logic is to be judged by continued activity surrounding open problems, then relevance logic must be accounted a flourishing area!

The relevance logic tradition, starting with Ackermann and continuing with the work of Alan Ross Anderson, Nuel D. Belnap and their students, has nurtured a fascinating collection of open problems that have stimulated research in the area for decades. Anderson provided an early collection of open problems in a paper [3] from 1963. The first in his list of problems is that of showing that the rule $\gamma$ (from $A$ and $\neg A \vee B$ to infer $B$ ) is admissible in $\mathbf{E}$. The second problem is that of finding a decision procedure for $\mathbf{E}$. The last problem is that of finding appropriate semantics for the whole of $\mathbf{E}$, with an appropriate completeness theorem. All of these problems were subsequently solved.

In addition, the two volumes of Entailment [1,2] contain a rich selection of open problems. Many of these problems have been solved subsequently due to the hard work of numerous researchers.

\footnotetext{
${ }^{*}$ The author gratefully acknowledges the support of the Natural Sciences and Engineering Research Council of Canada.
}

Australasian Journal of Logic (13:1) 2016, Article no. 2 


\section{Decision problem for the semilattice system}

A semantics based on semilattice models [21] was published by the author in 1972. The language for this semantics is $\rightarrow, \wedge, \vee$, that is to say, the language of the positive relevance logic $\mathbf{R}$. Let $\langle S, \cup, 0\rangle$ be a semilattice with zero. Assign propositional variables $P$ subsets of $S$ as values, written $V(P)$. Then the truth definition relative to a point $x$ in $S$ is defined inductively as follows:

1. $x \models P \Leftrightarrow x \in V(P)$, where $P$ is a variable;

2. $x \models A \wedge B \Leftrightarrow x \models A$ and $x \models B$;

3. $x \models A \vee B \Leftrightarrow x \models A$ or $x \models B$;

4. $x \models A \rightarrow B \Leftrightarrow \forall y[y \models A \Rightarrow x \cup y \models B]$;

A formula $A$ of positive relevance logic is valid in this semantics if $0 \models A$ for every assignment to its variables in a semilattice with zero.

This semantics validates all of the formulas of positive $\mathbf{R}$. When I first discovered the semantics in 1971, I thought that it was complete for this system. However, Robert K. Meyer and J.Michael Dunn soon discovered a formula that is valid for this semantics, but not provable in positive $\mathbf{R}$. I presented a slightly simplified version of their formula, namely

$$
[(A \rightarrow(B \vee C)) \wedge(B \rightarrow D)] \rightarrow(A \rightarrow(D \vee C))
$$

in my paper of 1972 .

Although the semilattice system $\mathbf{S}$ does not coincide with positive $\mathbf{R}$, contrary to my initial hopes, it is a very natural logic that has a good claim to be considered the most natural extension of implicational $\mathbf{R}$ to include conjunction and disjunction.

The semilattice system $\mathbf{S}$ proved somewhat tricky to axiomatize. The problem of axiomatization was solved by Kit Fine, who published his solution as an abstract [8]. Fine's axiomatization includes a rather complicated rule; a detailed version[5] of his completeness proof was published by Gerald Charlwood in 1981.

The axiomatic version of the semilattice system appears rather complicated and artificial. There are, however, two alternative ways to present the system that make it appear much more natural. The first way is to use an extension of the Fitch-style natural deduction system of Anderson and Belnap; this extension uses a strengthened version of the disjunction rules presented in the first volume of Entailment [1]. This new version of the natural deduction system is presented in my paper of 1989 [24].

In the original natural deduction rules of Anderson and Belnap, the distribution axiom does not follow from the introduction and elimination rules for conjunction and disjunction but has to be "put in by hand." The original rule of $\vee$ elimination $[1$, p. 272] is as follows:

Australasian Journal of Logic (13:1) 2016, Article no. 2 


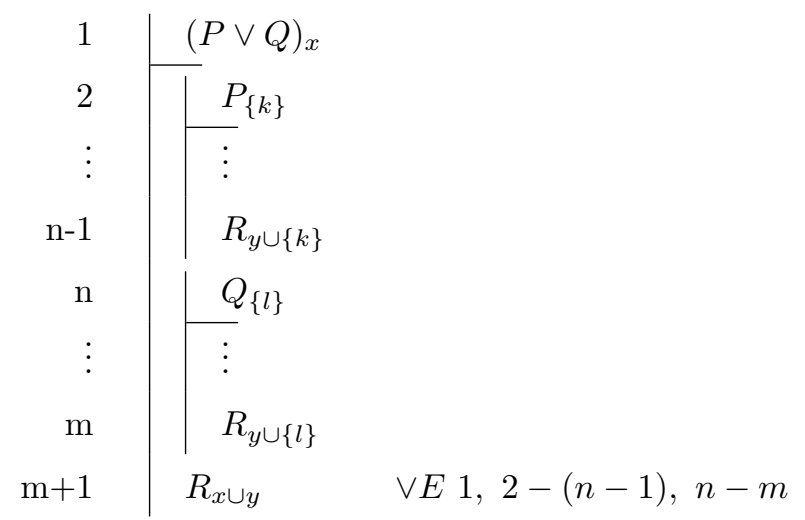

The new rule of $\vee$ elimination is as follows:

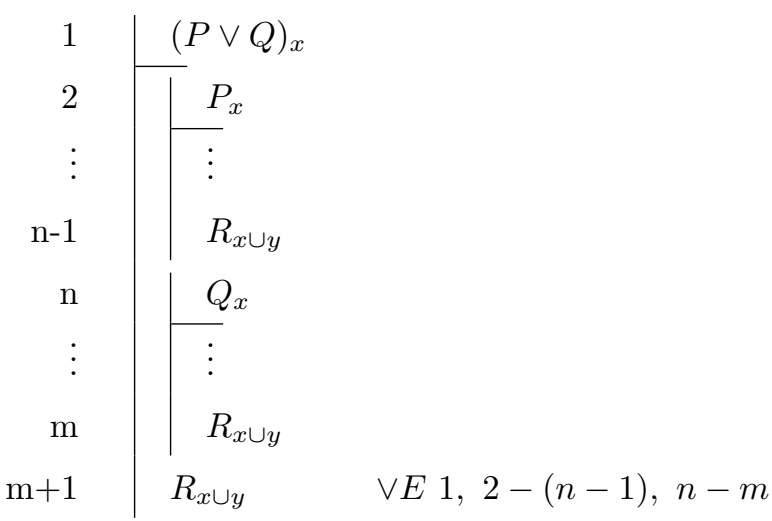

With this new natural deduction rule for disjunction, the distribution axiom follows unproblematically from the introduction and elimination rules.

Distribution is missing from the system [9] of linear logic, for much the same reasons that it is absent from the natural deduction system for relevance logic based only on the original introduction and elimination rules. Given the motivation of Anderson and Belnap, the distribution axiom should hold for $\wedge$ and $\vee$ - whether it should hold for the additive connectives of linear logic is much more doubtful, given the radically different motivation.

The fact that this system is a very natural extension of the pure theory of relevant implication appears also from the fact that it coincides with the system of positive relevant implication defined by a set of rules given by Dag Prawitz in Chapter VII of his well known monograph [16]. The equivalence of this system with the positive semilattice system is proved in detail by Charlwood in his thesis [4] of 1978.

Although one can make a good case that the positive semilattice system is the most natural extension of pure relevant implication (Church's theory of weak implication [6]), it has not been thoroughly investigated, in contrast to 
the better known logics of Ackermann, Anderson and Belnap. In particular, the decision problem seems to be wide open.

Problem 2.1 Is the positive semilattice system $\mathbf{S}$ decidable?

Although this problem appears to be open, one can make various remarks about it. First, the undecidability proofs for $\mathbf{R}, \mathbf{E}$ and other classical systems of relevance logics do not seem to adapt to the positive semilattice system. This is because the Meyer-Dunn formula above is not valid in the models constructed from projective spaces that are used in the undecidability proof. Second, if it is indeed decidable (and I venture the conjecture that it is), then the decision procedure cannot be primitive recursive. This follows from the fact that the implication-conjunction fragment of the system is the same as that of $\mathbf{R}$. In a paper of 1999 [26], I showed that there is no primitive recursive decision procedure for this fragment.

The decision problem for $\mathbf{R}_{\rightarrow}$ was solved by Saul Kripke [11] in 1959. It was extended to all of $\mathbf{L R}$ ( $\mathbf{R}$ without the distribution axiom) by Robert K. Meyer in his doctoral thesis [14]; an exposition of his decidability proof can be found in the monograph [19] by Thistlewaite, McRobbie and Meyer. Kripke's decision procedure is based on a cutfree sequent system for $\mathbf{R}_{\rightarrow}$, together with a lemma that is equivalent to Dickson's Lemma in the theory of polynomial ideals $[1$, pp. 138-139]. If we could extend the cutfree sequent system to $\mathbf{S}$, then we might be able to extend the decision procedure as well. Unfortunately, at the moment, the only cutfree system known for $\mathbf{S}$ uses subscripted formulas and a decision procedure based on this subscripted system is not known. A purely implicational version of the subscripted sequent system can be found in my paper [20]; a version for all of $\mathbf{S}$ is in my doctoral thesis [22].

The basic objects are sequents $\Gamma \vdash \Delta$, where $\Gamma$ and $\Delta$ are finite sets of subscripted formulas $A_{x}$, where $x$ is a finite set of positive integers. The subscripted sequent system for $\mathbf{S}$ has as axioms all sequents of the form

$$
\Gamma, A_{x} \vdash A_{x}, \Delta .
$$

The rules of inference are as follows:

$$
\begin{array}{cl}
\frac{\Gamma \vdash A_{y}, \Delta \quad B_{x \cup y}, \Theta \vdash \Lambda}{\Gamma, \Theta, A \rightarrow B_{x} \vdash \Delta, \Lambda}(\rightarrow \vdash) & \frac{\Gamma, A_{\{k\}} \vdash B_{x \cup\{k\}}, \Delta}{\Gamma, \vdash A \rightarrow B_{x}, \Delta}(\vdash \rightarrow) \\
\frac{\Gamma \vdash \Delta, A_{x} \Theta \vdash B_{x}, \Lambda}{\Gamma, \Theta \vdash A \wedge B_{x}, \Delta, \Lambda}(\wedge \vdash) & \frac{\Gamma, A_{x}, B_{x} \vdash \Delta}{\Gamma, A \wedge B_{x} \vdash \Delta}(\vdash \wedge) \\
\frac{\Gamma, A_{x} \vdash \Delta, \quad \Theta, B_{x} \vdash \Lambda}{\Gamma, \Theta, A \vee B_{x} \vdash \Delta, \Lambda}(\vee \vdash) & \frac{\Gamma \vdash A_{x}, B_{x}, \Delta}{\Gamma, \vdash A \vee B_{x}, \Delta}(\vdash \vee)
\end{array}
$$

Australasian Journal of Logic (13:1) 2016, Article no. 2 
In the rule $(\vdash \rightarrow)$, the numeral $k$ must not appear in the conclusion of the inference.

It is not too hard to show that the theorems of this subscripted sequent system coincide with those of $\mathbf{S}$, in the sense that the sequent $\vdash A_{\emptyset}$ is provable in the sequent system if and only if $A$ is provable in $\mathbf{S}$.

Problem 2.2 Can we base decision procedures on the subscripted sequent systems?

\section{$3 \quad$ Number of variables for undecidability}

I proved in the early 1980s [23] that $\mathbf{R}, \mathbf{E}$ and a large family of related relevance logics are undecidable. In my 1984 paper, I sketched a proof that the five variable fragment of $\mathbf{R}$ is undecidable. Unfortunately, there is a mistake in the proof given there, as I report in a paper [27] published in 2007. The claim, however, is correct (though the proof is in error) and I was able to show in the later paper that the four variable fragment of $\mathbf{R}$ is undecidable. This brings me to my third problem.

Problem 3.1 What is the smallest number of variables for which the corresponding fragment of $\mathbf{R}$ is undecidable?

To set the problem in its proper context, let us recall an outline of the undecidability proof for $\mathbf{R}$. The construction works by adapting a geometrical definition of multiplication given by von Staudt and employed by von Neumann in his work on continuous geometries [15].

Figure 1 illustrates the multiplication of two points on a line in projective space. The fixed coordinate frame is shown in black, while the red points can be considered as varying over the points in the frame. The points $a_{1}, a_{2}, a_{3}$ are the three points of the frame, while the auxiliary points $c_{12}, c_{23}, c_{13}$ serve as centres of perspectivity. Given points $x$ and $y$ on the line $L_{12}$ joining $a_{1}$ and $a_{2}$, we draw the line joining $x$ and $c_{23}$, and then find its point of intersection with the line joining $a_{1}$ and $a_{3}$. Similarly, we draw the line joining $y$ and $c_{13}$ and then find its point of intersection with the line joining $a_{2}$ and $a_{3}$. Then draw the line joining these two points of intersection; the point where this line intersects the base line joining $a_{1}$ and $a_{2}$ is the point $x \cdot y$. If we assume Desargues's law, then the geometrical multiplication defined in this way is associative.

In a two-dimensional projective space, however, we cannot assume the Desargues law in general, because of the existence of non-Arguesian projective planes. If we add a third dimension to our coordinate frame, however, then we can prove enough of Desargues's law to prove associativity of $x \cdot y$ with appropriate assumptions. This is the construction that proves undecidability for a wide family of relevance logics. Figure 2 shows a three-dimensional coordinate frame (a 4-frame). It consists of a tetrahedron, together with the auxiliary points forming the points of intersection with a skew plane cutting through the tetrahedron.

Australasian Journal of Logic (13:1) 2016, Article no. 2 


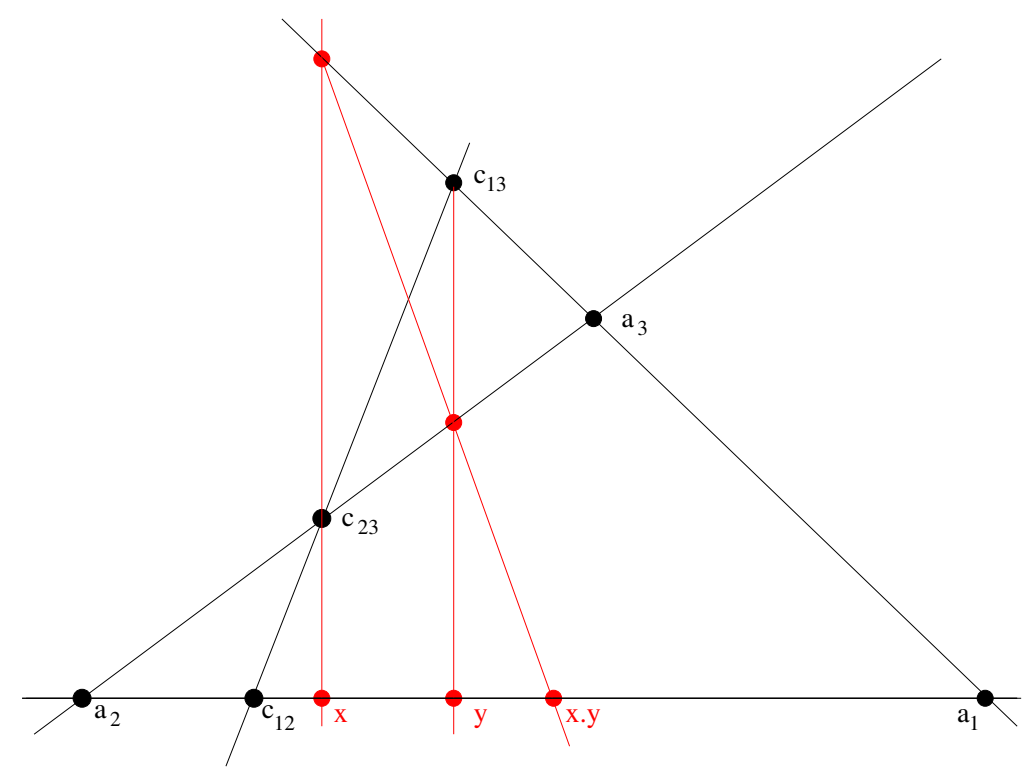

Figure 1: Multiplication on a line in real projective space

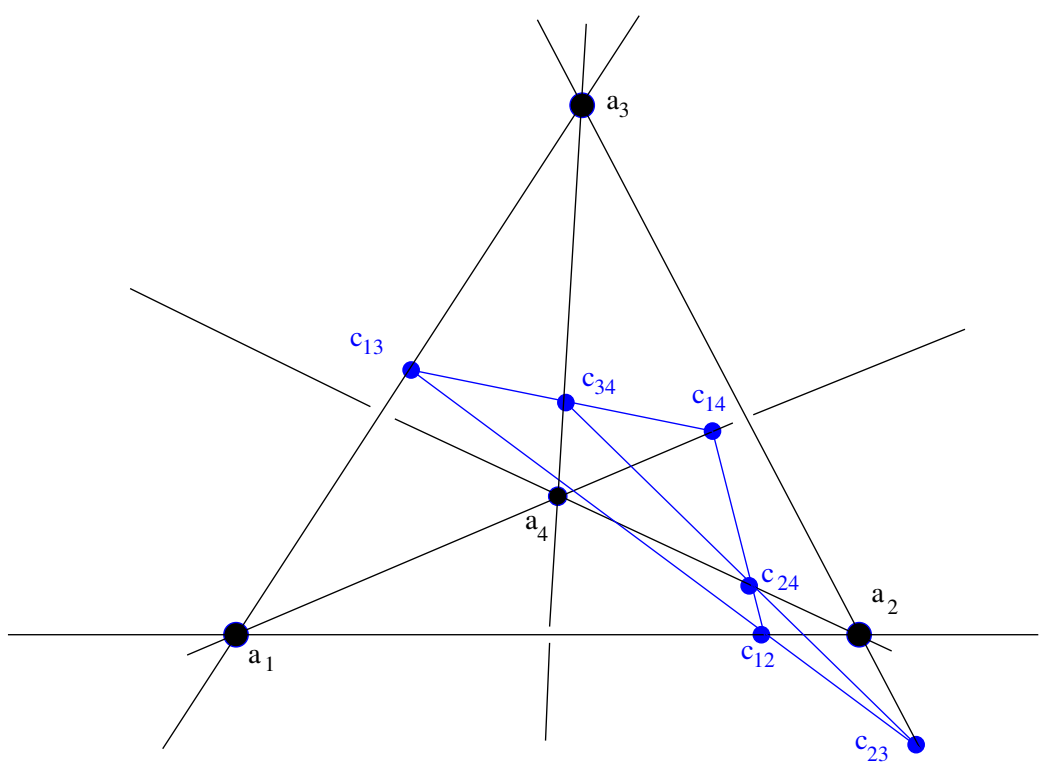

Figure 2: A 4-frame in real projective space

Australasian Journal of Logic (13:1) 2016, Article no. 2 
How do we reduce the number of variables required for undecidability? The trick is to express the elements in a coordinate frame in terms of subsets of the frame. For example, to prove undecidability for the four-variable fragment, we have to find a way to express the elements of a four-frame, together with semigroup elements $x, y$ in terms of four elements definable from the frame. The details can be found in my 2007 paper [27].

We can add the propositional constants $t, f, T, F$ to $\mathbf{R}$, and so it makes sense to talk of the 0 -variable fragment of $\mathbf{R}$. This fragment is in fact decidable. This follows from the proof by John Slaney [18] that there are exactly 3088 non-equivalent formulas generated from the constants in $\mathbf{R}$. This result forms a contrast with linear logic, where the fragment generated by the constants is undecidable, a remarkable result of Max Kanovich [10].

So, is the answer to problem 3.1 one, two, three or four? It is possible that the geometrical technique sketched above can be improved to prove undecidability for three variables. It seems implausible that it could be extended to two. As for the one-variable fragment, it remains deeply mysterious.

Thistlewaite, McRobbie and Meyer in their 1988 monograph [19, p. 16] suggested a way of proving undecidability for the two-variable fragment of $\mathbf{R}$. Let $x \odot y$ be a formula of $\mathbf{R}$ containing only the two variables $x$ and $y$. If $\sigma$ is a term in the language of semigroups, let $\sigma^{t}$ be the translation of $\sigma$ into $\mathbf{R}$, using the translation $x \cdot y \mapsto x \odot y$; the translation of a semigroup equality $\sigma=\tau$ is $\sigma^{t} \leftrightarrow \tau^{t}$. Let us say that the formula $\odot$ is a free associative connective in $\mathbf{R}$ if the following holds. If $\Sigma \cup\{\tau\}$ is a finite set of semigroup equalities, then $\tau$ is deducible from $\Sigma$ in the equational theory of semigroups if and only if $\tau^{t}$ is deducible from $\Sigma^{t}$ in $\mathbf{R}$.

Let us suppose that there is in fact a free associative connective in $\mathbf{R}$. Since there is a finitely presented semigroup in two generators with undecidable word problem, undecidability for formulas with two variables in $\mathbf{R}$ would follow immediately.

Problem 3.2 Is there a free associative connective in $\mathbf{R}$ ?

Somehow, I am inclined to guess that there is no such connective. The encoding of semigroup equations in the undecidability proofs is rather indirect, since the associativity is proved only with respect to a coordinate frame, employing variables additional to those used in encoding the semigroup equations. However if the preceding problem had a positive solution, associativity would have to hold unconditionally.

\section{Complexity of the decision problem for $R_{\rightarrow}$}

As mentioned above in $\S 2$, the decision problem for $\mathbf{R}_{\rightarrow}$ was solved by Saul Kripke in 1959. Kripke's decision procedure appears of high complexity - in fact, the algorithm does not appear to provide an upper bound on the space or time required. The key combinatorial lemma in the proof of correctness is dubbed "Kripke's Lemma" by Anderson and Belnap [1, pp. 138-139]; it is essentially 
the same as Dickson's Lemma in number theory and the theory of polynomial ideals. By adapting known bounds for Dickson's Lemma and related problems, it is possible to show [26] Kripke's decision method is primitive recursive in the Ackermann function.

Can we improve on this Ackermann upper bound by getting a tighter estimate of the complexity? I made a small step in this direction by proving that any decision procedure for $\mathbf{R}_{\rightarrow}$ requires exponential space. This was published in a 1990 paper [25] as part of a festschrift in honour of my Doktorvater, Nuel Belnap. The basic idea is to adapt the exponential space lower bound for the reachability problem for Petri nets proved by R.J. Lipton [13].

The lower bound in both cases is proved by encoding bounded counter machines in the system, and depends on the fact that we can define small (linearsize) vector addition systems that generate a doubly exponential number of tokens. This allows the definition of a zero-test for counter machines in which the numbers in the counters are exponentially bounded. Some added complications in the logical case arise from the fact that the unrestricted contraction rule is present.

This still leaves a huge gap between the upper and the lower bounds. I was able to close the gap a few years later, in the case where we include conjunction as well as implication. The same upper bound holds as in the case of pure relevant implication. But in addition, in a paper of 1999 [26] I was able to prove that the lower and upper bounds for the system $\mathbf{R}_{\rightarrow \wedge}$ essentially coincide, showing that there is no primitive recursive decision procedure for this logic. Thus $\mathbf{R}_{\rightarrow \wedge}$ is one of the most complex naturally defined propositional logics.

The lower bound for $\mathbf{R}_{\rightarrow \wedge}$ is an adaptation of the proof by Lincoln, Mitchell, Scedrov and Shankar [12] that linear logic is undecidable. Unfortunately, the proof does not adapt to the pure implicational case, since it depends on the inclusion of additive as well as multiplicative rules. I made several efforts in the following decades to narrow the gap, but did not succeed. I was planning to present this as an open problem as part of my talk, but shortly before the July 2014 Summer of Logic in Vienna, I was delighted to hear that the problem has been definitely solved by Sylvain Schmitz.

Sylvain Schmitz proves that in fact the pure implicational fragment $\mathbf{R}_{\rightarrow}$ is complete for doubly exponential time, thus solving a problem that has been open for a quarter century. His proof [17] employs branching vector addition systems and builds on earlier results of Demri, Jurdziński, Lachish and Lazić [7]. This brilliant breakthrough result of Schmitz seems an appropriate point to bring my survey to a close. ${ }^{1}$

\section{References}

[1] A. R. Anderson and N. D. Belnap. Entailment, volume 1. Princeton University Press, Princeton, NJ, 1975.

\footnotetext{
${ }^{1}$ I thank the referee for useful comments that improved the exposition.
} 
[2] A. R. Anderson, N. D. Belnap Jr., and J. M. Dunn. Entailment, volume 2. Princeton University Press, Princeton, NJ, 1992.

[3] Alan Ross Anderson. Some open problems concerning the system E of entailment. In Proceedings of a Colloquium on Modal and Many-Valued Logics, Helsinki, 23-26 August, 1962, 1963. Acta Philosophica Fennica, Fasc. 16.

[4] G. Charlwood. Representations of semilattice relevance logic. PhD thesis, University of Toronto, 1978.

[5] G. Charlwood. An axiomatic version of positive semilattice relevance logic. Journal of Symbolic Logic, 46:233-239, 1981.

[6] Alonzo Church. The weak theory of implication. In Angsil Menne, Wilhelmy, editor, Kontrolliertes Denken, Untersuchungen zum Logikkalkül und der Logik der Einzelwissenschaften, pages 22-37. Kommissions-Verlag Karl Alber, 1951.

[7] Stéphane Demri, Marcin Jurdziński, Oded Lachish, and Ranko Lazić. The covering and boundedness problems for branching vector addition systems. Journal of Computer and System Sciences, 79:23-38, 2013.

[8] Kit Fine. Completeness for the semilattice semantics with disjunction and conjunction. Journal of Symbolic Logic, 41:560, 1976. Abstract.

[9] Jean-Yves Girard. Linear logic. Theoretical Computer Science, 50:1-102, 1987.

[10] Max Kanovich. Simulating linear logic in 1-only linear logic. Technical Report 94-02, CNRS, Laboratoire de Mathématiques Discrètes, January 1994.

[11] Saul A. Kripke. The problem of entailment. The Journal of Symbolic Logic, 24:324, 1959. Abstract.

[12] P. Lincoln, J. Mitchell, A. Scedrov, and N. Shankar. Decision problems for propositional linear logic. Annals of Pure and Applied Logic, 56:239-311, 1992.

[13] R.J. Lipton. The reachability problem requires exponential space. Technical Report 62, Yale University, 1976.

[14] Robert.K. Meyer. Topics in Modal and Many-valued Logic. PhD thesis, University of Pittsburgh, Pennsylvania, 1966.

[15] John von Neumann. Continuous Geometry. Princeton University Press, Princeton, New Jersey, 1960.

[16] Dag Prawitz. Natural Deduction. A Proof-theoretical study. Almqvist and Wiksell, 1965. Reprinted by Dover books 2006.

Australasian Journal of Logic (13:1) 2016, Article no. 2 
[17] Sylvain Schmitz. Implicational relevance logic is 2-Exptime-complete. arXiv:1402.0705v1 [cs.LO] 4 February 2014; forthcoming Journal of Symbolic Logic.

[18] John K. Slaney. 3088 varieties: A solution to the Ackermann constant problem. Journal of Symbolic Logic, pages 487-501, 1985.

[19] P.B. Thistlewaite, M.A. McRobbie, and R.K. Meyer. Automated theoremproving in non-classical logics. Pitman, London, 1988.

[20] Alasdair Urquhart. Completeness of weak implication. Theoria, 37:274282, 1971.

[21] Alasdair Urquhart. Semantics for relevant logics. Journal of Symbolic Logic, 37:159-169, 1972.

[22] Alasdair Urquhart. The Semantics of Entailment. PhD thesis, University of Pittsburgh, $1973 . \quad$ Available at https://utoronto academia.edu/AlasdairUrquhart/Thesis.

[23] Alasdair Urquhart. The undecidability of entailment and relevant implication. Journal of Symbolic Logic, 49:1059-1073, 1984.

[24] Alasdair Urquhart. What is relevant implication? In Norman and Sylvan, editors, Directions in Relevant Logic, pages 167-74. Kluwer, 1989.

[25] Alasdair Urquhart. The complexity of decision procedures in relevance logic. In J. Michael Dunn and Anil Gupta, editors, Truth or Consequences: Essays in honour of Nuel Belnap, pages 61-76. Kluwer, Dordrecht, 1990.

[26] Alasdair Urquhart. The complexity of decision procedures in relevance logic II. Journal of Symbolic Logic, 64:1774-1802, 1999.

[27] Alasdair Urquhart. Four variables suffice. Australasian Journal of Logic, 5:66-73, 2007. 